\title{
Efeitos do desalinhamento da taxa real de câmbio sobre o crescimento econômico*
}

\author{
Effects of the Real Exchange Rate Misalignment on Economic Growth
}

\author{
Kerssia Preda Kamenach, Sérgio Fornazier Meyrelles Filho, \\ Fabrício José Missio e Stefan Wilson D’Amato**
}

\begin{abstract}
Resumo: O objetivo do artigo é analisar empiricamente os efeitos do desalinhamento da taxa real de câmbio sobre o crescimento econômico. Para tanto, por meio da metodologia de dados em painel, testamos essa relação econometricamente para uma amostra de 96 países no período de 2001 a 2014. A novidade do trabalho está na amostra, no período analisado e na especificação das variáveis de controle, o que inclui o índice de complexidade econômica (ICE). Esse índice é utilizado como uma proxy para o nível de desenvolvimento tecnológico. Os resultados indicam que a manutenção de uma taxa real de câmbio subvalorizada afeta positivamente o crescimento econômico, especialmente para países em desenvolvimento.
\end{abstract}

Palavras-chave: Desalinhamento cambial; Taxa real de câmbio e crescimento econômico

Abstract: The aim of the article is to empirically analyze the effects of exchange rate misalignment on economic growth. Therefore, using the panel data methodology, we tested this relationship econometrically for a sample of 96 countries from 2001 to 2014. Our contribution is the use of a different dataset that increases the number of countries in the sample, as well as the control variables which includes the economic complexity index (ICE). This index is used as a proxy for the level of technological development. The results indicate that competitive real exchange rate management affects positively the rate of output growth, especially for developing countries.

Keywords: Exchange misalignment; Real exchange rate and economic growth

JEL: F41; F43; F49

\footnotetext{
* Submissão: 27/02/2020 | Aprovação: 21/08/2020 | DOI: 10.5380/re.v42i78.71313

** Respectivamente: (1) Programa de Pós-Graduação em Economia da Universidade Federal de Goiás (PPGECON/UFG) |E-mail: kerssia.kamenach@ @mail.com |ORCID: 0000-0003-3140-2873 | (2) Professor Associado da Universidade Federal de Goiás. Programa de Pós-Graduação em Economia (PPGECON/UFG) | E-mail: fornazier@ufg.br | ORCID: 0000-0002-1978-789X | (3) Professor do Centro de Desenvolvimento e Planejamento Regional da Universidade Federal de Minas Gerais (CEDEPLAR/UFMG) | E-mail: fjmissio@ cedeplar.ufmg.br | ORCID: 0000-0003-4561-6039 | (4) Doutorando em Economia pelo Centro de Desenvolvimento e Planejamento Regional da Universidade Federal de Minas Gerais (CEDEPLAR/UFMG) | E-mail: swdamato@ cedeplar.ufmg.br | ORCID: 00000003-4159-0396
} 


\section{Introdução}

Estudos empíricos sobre os determinantes do desenvolvimento econômico tendem a se concentrar no papel desempenhado pela acumulação de capital físico e em fatores como educação, produtividade total dos fatores e grau de desenvolvimento institucional. Essas dimensões são, via de regra, apontadas como as causas fundamentais do crescimento econômico.

Não obstante, é crescente a literatura que analisa o papel de outras variáveis enquanto determinantes do crescimento como, por exemplo, aquela que analisa $o$ papel da taxa real de câmbio (RER) (Barbosa-Filho, 2006; Rodrik, 2008; Blecker, 2016; Bresser-Pereira et al., 2017). Destacam-se os estudos que analisam os efeitos do desalinhamento cambial sobre as taxas de crescimento dos países, considerados seus distintos graus de desenvolvimento (Gala, 2008; Freund; Pierola, 2012; Cimoli et al., 2013; Missio et al. 2015; Gabriel et al., 2016).

Conceitualmente, o desalinhamento cambial ocorre quando a RER observada difere do seu valor de equilíbrio de longo prazo, definido este a partir de "fundamentos" econômicos. Nurkse (1945) definiu a taxa de câmbio de equilíbrio de longo prazo como sendo o valor que garante simultaneamente o equilíbrio interno e externo, situação na qual a economia opera em condições de pleno emprego da força de trabalho. Para Montiel (1999), trata-se do nível da taxa real de câmbio para o qual as expectativas a respeito do valor futuro não têm influência na determinação da variável em consideração. Ou ainda, a despeito da inexistência de um consenso na literatura quanto à definição mais apropriada do referido equilíbrio, convencionalmente, caracteriza-se uma situação de subvalorização cambial quando a taxa real de câmbio encontra-se abaixo de seu equilíbrio de longo prazo (Oreiro et al., 2011).

Nesse contexto, o presente estudo tem por objetivo investigar empiricamente os efeitos do desalinhamento da taxa real de câmbio sobre o crescimento econômico. Utiliza-se o índice de desalinhamento - calculado como sendo a taxa real de câmbio ajustada pelo efeito Balassa-Samuelson desenvolvido por Rodrik (2008). Para tanto, estima-se um modelo econométrico em dados em painel, com base em uma amostra de 96 países formada por economias avançadas e por economias em desenvolvimento no período de 2001 a 2014. Além disso, o exercício controla a taxa de crescimento pelo grau de desenvolvimento tecnológico dos países, de forma a captar de maneira mais 
precisa a relação de interesse. Utiliza-se, para tanto, o Índice de Complexidade Econômica (ICE) proposto por Hausmann e Hidalgo (2011).

$\mathrm{O}$ artigo contribui com a literatura na medida em que fornece evidências empíricas que, ainda que não sejam totalmente inovadoras, são importantes para validar certas construções teóricas e, mais, porque podem ser vistas como testes de robustez para determinados achados empíricos. Ademais, o trabalho inova ao utilizar uma amostra diferente de países e ao empregar o ICE como variável de controle.

O trabalho encontra-se dividido em três seções, além desta introdução e das considerações finais. A seção dois apresenta o marco analítico sobre o tema; a seção seguinte sistematiza os aspectos metodológicos gerais da análise proposta, incluindo questões relativas aos dados utilizados e à especificação econométrica empregada; a seção quatro apresenta e analisa os resultados alcançados.

\section{Marco analítico}

Existe uma crescente literatura dedicada a analisar os potenciais efeitos de longo prazo do nível da taxa real de câmbio sobre o processo de crescimento. Por exemplo, na abordagem dos modelos de crescimento puxado pelas exportações (export-led), essa variável é apontada como capaz de induzir uma mudança estrutural em direção a setores mais sofisticados ${ }^{1}$. Nesse sentido, ela tem efeitos duradouros sobre a taxa de crescimento do produto, uma vez que a nova composição setorial tende a aumentar a produtividade geral da economia.

A relação positiva entre a taxa de crescimento da produtividade do trabalho e a participação do setor industrial no produto é um fato estilizado com robustez empírica (McCombie et. al., 2009; Romero; Britto, 2016). Em grande parte, essa relação justifica-se pelo fato de que o setor exibe retornos dinâmicos de escala, maior acumulação de capital e é particularmente propício à existência de spillovers tecnológicos (Kaldor, 1967, 1970).

De forma semelhante, na abordagem dos modelos de crescimento com equilíbrio intertemporal do Balanço de Pagamentos (restrição externa), uma série de estudos recentes têm demonstrado a relevância do nível da RER no crescimento

\footnotetext{
${ }^{1}$ Mudança estrutural consiste na transferência de recursos entre os setores. Admite-se que a mudança é prócrescimento quando ela transfere recursos de setores tradicionais ou agrícolas para setores modernos ou manufatureiros/industriais. Para maiores informações ver: Lewis (1954), Clark (1967), Kaldor (1966), Kuznets (1966; 1979), Denison (1967), Syrquin (1988), Meyer (2008), McMillan e Rodrik (2011), Vu (2017) e outros.
} 
econômico de longo prazo. Essa literatura mostra que o gerenciamento da taxa de câmbio real, de forma a mantê-la estável e competitiva, é importante nesse processo porque, entre outros efeitos, tende a relaxar a restrição externa, viabilizando um crescimento mais robusto, especialmente no caso de países menos desenvolvidos, onde, em regra, por razões estruturais, a restrição externa manifesta-se de forma mais severa (Missio; Jayme Junior, 2012; Gouvea; Lima, 2013; Ferrari et al., 2013; Marconi et al., 2015; Oreiro et al., 2015; entre outros).

Interações entre a RER e outras variáveis econômicas (como o progresso tecnológico e a distribuição de renda, por exemplo) também tem se mostrado fundamentais ao crescimento (Razmi, 2015; Boggio; Barbieri, 2017; Martins Neto; Porcile, 2017; Ribeiro et al., 2020). Essas relações têm sido gradativamente estudadas tanto do ponto de vista teórico como empírico, caracterizando um marco analítico em contínuo processo de construção.

Eichengreen (2008) afirma que, além de os países sustentarem uma taxa de câmbio estável e competitiva, é necessário também desenvolver outras estratégias para promover o crescimento. Tais estratégias devem envolver elementos como a manutenção da estabilidade política, a melhoria no arcabouço institucional e o desenvolvimento do mercado financeiro. Além disso, o autor argumenta que a subvalorização deve ser mantida apenas por um prazo limitado, caso contrário, essa política pode ter efeitos estruturais deletérios para a economia.

Segundo Rodrik (2008), a manutenção de uma taxa de câmbio competitiva tem efeitos positivos sobre o crescimento na medida em que induz a mudança estrutural em direção a setores tradables. Esses setores sofrem menos com as falhas institucionais e de mercado, que são características presentes principalmente nos países em desenvolvimento. Além disso, na medida em que a subvalorização aumenta a rentabilidade, ela induz a expansão nos investimentos e melhorias em termos da eficiência produtiva.

Para Glüzmann et al. (2012), uma taxa de câmbio real depreciada promove o crescimento, pois aumenta o investimento e o emprego. Os autores sugerem que os canais de transmissão atuam via mudança estrutural ${ }^{2}$ e via redução dos custos com a mão de obra, que tem sua contrapartida no aumento dos fundos internos das empresas. Isso é particularmente importante para as empresas em países em

\footnotetext{
${ }^{2}$ Com a transferência de trabalhadores dos setores agrícolas (tradicional) de subsistência, com baixa produtividade, para setores industriais de alta produtividade (modernos).
} 
desenvolvimento que tendem a sofrer com restrições financeiras e de acesso ao crédito. Eles identificam, ainda, impactos diferenciados da taxa de câmbio real nos diferentes setores da economia.

Frenkel e Rapetti (2014) argumentam que a taxa de câmbio real deve ser estável e subvalorizada a fim de estimular a comercialização de bens com maior intensidade tecnológica. Nessa mesma linha de raciocínio, Gabriel e Missio (2018) mostram que existe uma inter-relação entre RER e o nível de complexidade econômica. A análise desenvolvida mostra que, ao afetar a participação da indústria no produto e, portanto, aumentar a complexidade da economia, a RER exerce influência importante sobre as taxas de crescimento dos países em desenvolvimento.

Em resumo, do ponto de vista teórico, tem surgido uma série de trabalhos mostrando os efeitos diretos da RER sobre a taxa de crescimento da economia, bem como os efeitos indiretos em outras variáveis, como a distribuição de renda, o progresso tecnológico, entre outras. Ressalta-se que os mecanismos de transmissão operam tanto pelo lado da demanda quanto da oferta. São exemplos os seguintes trabalhos: a) sobre a taxa de crescimento das economias (Marconi $e t$ al., 2015; Missio et al., 2015; Guzman et al., 2018); b) sobre a estrutura produtiva (Ahmed et al., 2016; Gabriel et al., 2016; Missio et al., 2017; Vu, 2017); c) sobre o progresso tecnológico/complexidade econômica (Chen, 2017; Gabriel; Missio, 2018); d) sobre a distribuição da renda (Ribeiro et al., 2020), entre outros.

Existe também uma crescente literatura dedicada a avaliar empiricamente os efeitos da RER sobre a taxa de crescimento do produto. Rodrik (2008) analisa econometricamente um conjunto de 188 países no período entre 1950-2004 e conclui que depreciações sustentadas na taxa de câmbio real geram um aumento na lucratividade relativa dos investimentos em bens comercializáveis (tradables). Isso, por sua vez, estimula o crescimento econômico de países em desenvolvimento. Os autores Di Nino, Eichengreen, Sbracia (2013) encontram resultados semelhantes a partir de modelos de painel (OLS e GMM) para uma amostra de 180 países no período de 1950-2009. Já Habib, Mileva e Stracca (2017) desenvolvem um estudo empírico a partir de modelos de dados em painel empregando variáveis instrumentais para 150 países no período entre 1970-2010. Os resultados mostram que a depreciação (apreciação) da taxa de câmbio impacta 
de forma positiva (negativa) no crescimento de economias em desenvolvimento e que os efeitos não são uniformes em termos setoriais.

São exemplos, também, os seguintes trabalhos empíricos: Baltar, Hiratuka e Lima (2016); El-Shagi, Lindner e Von Schweinitz, (2016); Sekkat (2016); Bordo et al. (2017); Habib, Mileva e Stracca (2017); Comunale (2017); Schroeder (2017), dentre outros.

Em resumo, pode-se dizer que o "estado da arte" dessa literatura incorpora três grandes movimentos: i) o primeiro, já em fase de superação, mostra a existência de uma relação positiva entre a manutenção de uma taxa de câmbio real competitiva e a taxa de crescimento do produto; ii) o segundo, em fase de consolidação e em decorrência do primeiro, busca identificar por meio de quais mecanismos a RER afeta a economia - nesse caso, o movimento é muito mais amplo, pois não se restringe aos efeitos sobre as taxas de crescimento; e, iii) a agenda empírica, a partir da incorporação das mais variadas formas e técnicas econométricas.

De maneira geral, os seguintes resultados (especialmente para os países em desenvolvimento) são respaldados pela evidência empírica: i) a apreciação cambial tem efeitos negativos sobre o crescimento; ii) o efeito da desvalorização cambial, em termos agregados, oscila entre neutro e positivo a depender do método e da amostra utilizada; iii) os efeitos da desvalorização diferem entre os setores (e intrasetorialmente); e iv) os efeitos do câmbio sobre o crescimento econômico podem ser não lineares.

O exercício realizado a seguir se enquadra dentro do primeiro movimento citado anteriormente. Entende-se que a sua realização é importante pois avança na consolidação empírica de uma relação econômica que é o fio condutor de toda uma agenda de pesquisa que nacionalmente e internacionalmente tem crescido e despertado a atenção dos policymakers.

\section{Procedimentos metodológicos}

\subsection{Abordagem econométrica}

A estrutura dos dados é formada por múltiplas unidades de cross-section (países) observadas ao longo do tempo (dimensão longitudinal), configurando, portanto, um painel. 
A Tabela 2 (apêndice) apresenta os resultados dos testes para a escolha do modelo apropriado. Primeiro, testou-se a presença de efeitos fixos. Nesse caso, realizou-se o teste F, pelo qual ficou rejeitada a hipótese nula de que os erros idiossincráticos são independentes e identicamente distribuídos, o que permite concluir que o modelo de efeitos fixos apresenta-se como mais apropriado do que o modelo MQO com dados empilhados. Em seguida, testou-se a presença de efeitos aleatórios, por meio do teste multiplicador de Lagrange proposto por Breusch e Pagan (1980), que indica a presença de efeitos aleatórios. Por fim, para escolher entre os efeitos fixos e aleatórios, utilizou-se o teste de Hausman (1978). Os resultados mostram que a hipótese nula, de que os coeficientes são não sistemáticos, é rejeitada para ambas as amostras, de forma a concluir que o modelo de efeitos fixos é o mais adequado. Por fim, vale ressaltar que, para fins de comparação, serão apresentadas também estimativas com pooled OLS e as estimativas do modelo de dados em painel com efeitos aleatórios.

A partir dos dados, compõe-se duas amostras: i) amostra ampla (96 países, sendo 31 economias avançadas e 65 em desenvolvimento); e, ii) amostra restrita (65 países em desenvolvimento). O período compreende os anos de 2001 a 2014. Os dados de todas unidades estão disponíveis ao longo de todo o período de tempo (painel balanceado). O recorte amostral e temporal segue a disponibilidade dos dados, especialmente da Penn World Table (versão 9.0), que disponibiliza informações relacionadas a 182 países entre 1950 e 2014. Nesse caso, o recorte da amostra e do período seguiram os seguintes critérios: i) montar um painel balanceado; e ii) verificar se a relação de interesse é válida no período recente. Nesse caso, nosso interesse está na análise do papel da RER sobre a taxa de crescimento considerando o período mais próximo da atualidade possível, dado a disponibilidade dos dados. Ademais, optamos por começar a análise a partir do ano de 2001 por duas razões: i) porque a segunda metade da década de 1990 houve uma série de crises cambiais, o que implica em dificuldades adicionais no tratamento econométrico (ao nosso ver, desnecessárias); e ii) porque, a partir desse período, houve um substancial aumento no número de países que adotaram o regime de câmbio flutuante (FMI, 2018). Então, o objetivo foi construir uma amostra mais homogênea, de forma a captar da melhor forma possível a relação de interesse.

Por fim, o exercício empírico realizado segue a literatura recente sobre o tema (Rodrik, 2008; Araújo; Gala, 2012). O primeiro passo é calcular o nível da 
taxa real de câmbio considerando o efeito Balassa-Samuelson ${ }^{3}$ e, com isso, seu índice de subvalorização. Em seguida, verificamos se esse índice tem efeitos sobre a taxa de crescimento do PIB per capita para um conjunto de países. A referida literatura não rejeita a hipótese de que países com relativa sobrevalorização do nível da taxa real de câmbio experimentam, em média, menores taxas de crescimento da renda per capita.

\subsection{Cálculo do Índice de Desalinhamento}

Seguimos a metodologia desenvolvida por Rodrik (2008), que é construída em três etapas. Em primeiro lugar, calcula-se a taxa real de câmbio utilizando dados Penn World Table 9.0;

$$
\ln R E R_{i t}=\ln \left(X R A T_{i t} / P P P_{i t}\right) .
$$

Na equação (1) $X R A T_{i t}$ é a taxa de câmbio nominal e $P P P_{i t}$ fator de conversão de paridade do poder de compra, ambos expressos como unidades monetárias nacionais por dólar norte-americano; $i$ indica países, $t$ é o tempo e $l n$ é o logaritmo natural. Quando $R E R_{i t}$ é maior que a unidade, o valor da moeda corrente é menor (mais depreciada) que o valor indicado pela paridade do poder de compra.

Em segundo lugar, ajusta-se a taxa real de câmbio calculada pelo efeito Balassa-Samuelson, ou seja, a equação (1) precisa ser corrigida pelas diferenças nas dotações de fatores, sendo o Produto Interno Bruto per capita em dólares (pibpcd) uma variável proxy para essa dotação. Logo, é preciso realizar o seguinte procedimento:

$$
\ln R E R_{i t}=\alpha+\beta \ln p i b p c_{i t}+f_{t}+\varepsilon_{i t},
$$

$f_{t}$ é o efeito fixo por período e $\varepsilon_{i t}$ é o termo de erro.

Finalmente, calcula-se o índice de desalinhamento, pela diferença entre a taxa real de câmbio e a taxa ajustada pelo efeito Balassa-Samuelson:

$$
\ln \left(U N D E R V A L_{i t}\right)=\ln R E R_{i t}-\ln \widehat{R E R_{l t}}
$$

\footnotetext{
${ }^{3}$ Segundo o efeito Balassa-Samuelson, o câmbio deve refletir o diferencial de produtividade entre os setores de bens comercializáveis e não comercializáveis com o exterior em cada país. Em outras palavras, um crescimento da produtividade dos bens tradables versus non-tradables de um país em relação aos países estrangeiros aumenta seu salário relativo, cresce o preço relativo dos non-tradables e, por conseguinte, o preço relativo médio, o que induz uma apreciação na taxa real de câmbio.
} 
em que $\widehat{R E R}_{i t}$ é o valor obtido pela equação (1).

Esse índice é comparável entre os países e ao longo do tempo. À medida que o índice de desalinhamento exceder a unidade, a moeda estará subvalorizada e, quando estiver abaixo da unidade, a moeda estará sobrevalorizada. Com base nesse índice, é possível investigar a relação entre desalinhamento da taxa real de câmbio e crescimento econômico.

\subsection{Modelo econométrico e variáveis utilizadas}

A forma geral da equação a ser estimada é dada a seguir, representando o modelo de crescimento para dados em painel:

$$
\text { TGROWTH } H_{i, t}=\beta_{0}+\beta_{2} \text { UNDERVAL }_{i, t}+\sum_{j=3}^{K} \beta_{j} Z_{i, t j}+\mu_{t}+\eta_{i}+\varepsilon_{i, t}
$$

Nesse modelo, a variável dependente é a taxa de crescimento (TGROWTH); $\beta_{s}^{\prime}$ são os parâmetros a serem estimados; UNDERVAL é o índice do desalinhamento da taxa real de câmbio calculado na equação (3); $Z$ são as variáveis de controle; $\mu$ é o efeito específico de tempo; $\eta$ captura os efeitos não observados de cada país nos quais são invariantes no tempo; $\varepsilon$ é o termo de erro idiossincrático; e os subscritos $i$ e $t$ referem-se a países e ao tempo, respectivamente; $i=$ $1, \ldots, N, t=2, \ldots, T, j=3, \ldots, K$.

As variáveis de controle utilizados para estimar o modelo da equação (4) seguem a literatura sobre o tema e podem ser classificadas nos seguintes grupos: (i) grau de abertura ao comércio internacional (OPENCTRADE) - que busca captar os ganhos advindos do comércio exterior, conforme estabelece a teoria do comércio internacional; ii) a taxa de inflação (INF) como proxy como indicação de estabilidade macroeconômica; iii) taxa de investimento (INVEST) - como proxy para a acumulação de capital físico, conforme destacado pela literatura de crescimento; iv) educação (EDUC) baseado em anos de escolaridade e retorno à educação; e v) grau de abertura financeira (KAOPEN).

Além disso, o Índice de Complexidade Econômica (ICE) será utilizado como uma variável explicativa, o que significa uma inovação em termos do exercício aqui proposto. Em termos teóricos, a inclusão desse índice é importante porque ele pode ser considerado uma proxy para a competitividade não preço da economia. Ou seja, o ICE capta a distância relativa dos sistemas produtivos à fronteira tecnológica. Logo, um ICE mais alto significa uma proximidade com a fronteira tecnológica 
mundial. Nesse sentido, esse índice capta a sofisticação produtiva que, por sua vez, é um importante determinante das taxas de crescimento econômico.

Vale lembrar que o índice de complexidade combina informações sobre complexidade e ubiquidade dos bens que um país produz para calcular uma medida de suas capacidades disponíveis. Países mais diversificados produzem bens menos ubíquos e países mais especializados produzem bens comuns. De acordo com Hausmann e Hidalgo (2011), o nível de complexidade dos bens produzidos e exportados afeta diretamente o processo de desenvolvimento econômico. Durante o processo de desenvolvimento, os países tendem a diversificar sua produção até alcançarem níveis de renda mais elevados. Portanto, é essencial controlar as estimativas por esses aspectos, ou seja, pelo grau de desenvolvimento e diversificação da estrutura produtiva.

Ademais, conforme visto no marco analítico, boa parte dos efeitos do RER sobre o crescimento econômico advêm dos seus efeitos sobre a competitividade não preço da economia. Sendo assim, quando usamos as duas variáveis - RER e ICE - como variáveis explicativas, então os coeficientes associados a variável de interesse (UNDERVAL) captam mais propriamente os efeitos dessa variável sobre o crescimento, que é o foco central do exercício proposto.

Em termos econométricos, a não inclusão dessa variável pode gerar estimativas inconsistentes. Ou seja, com base na literatura, sabemos que uma RER subvalorizada tem um impacto positivo na complexidade econômica dos países em desenvolvimento (GABRIEL; MISSIO, 2018). Ao mesmo tempo, existem trabalhos que mostram a importância do ICE sobre a taxa de crescimento desses países (BASTOS e WANG, 2015; DEMIRAL, 2016; STOJKOSKI et. al. 2017). Portanto, se o índice não for incluído como variável explicativa, ele estará no termo de erro. Nesse caso, tanto a variável dependente quanto a variável independente estarão correlacionadas com esse termo e, portanto, o modelo estará mal especificado.

A Tabela 1 a seguir sintetiza as variáveis utilizadas no exercício econométrico ${ }^{4}$ indica as respectivas fontes dos dados.

\footnotetext{
${ }^{4}$ Algumas estatísticas descritivas das variáveis utilizadas nos exercícios econométricos estão disponíveis no apêndice.
} 
Tabela 1 - Descrição e fonte dos dados

\begin{tabular}{c|l|c} 
Variável & \multicolumn{1}{|c}{ Característica } & Fonte \\
$T G R O W T H$ & Taxa de crescimento do PIB & WDI \\
\hline UNDERVAL & $\begin{array}{l}\text { Índice de desalinhamento da taxa real de } \\
\text { câmbio calculado segundo Rodrik (2008) }\end{array}$ & $\begin{array}{c}\text { Elaboração própria } \\
\text { com dados do WDI }\end{array}$ \\
\hline$I C E$ & Índice de complexidade & AEC/HUKSG \\
\hline EDUC & Educação & PWT 9.0 \\
\hline$I N F L A$ & $\begin{array}{l}\text { Taxa de inflação (Preço médio ao } \\
\text { consumidor) }\end{array}$ & WEO/IMF \\
\hline INVEST & Taxa de investimento & WEO/IMF \\
\hline OPENTRADE & $\begin{array}{l}\text { Soma das exportações e importações de } \\
\text { bens e serviços como parcela do PIB }\end{array}$ & WDI \\
\hline
\end{tabular}

Fonte: elaboração dos autores. Nota: WEO -World Economic; PWT - Pen World Table; AEC Atlas of Economic Compexity; HUKSG - Harvard University's Kennedy School of Government; WDI - World Development Indicators.

\section{Resultados e discussões}

Realizamos uma série de exercícios econométricos para investigar a relação entre as variáveis de interesse (desalinhamento taxa real de câmbio e crescimento econômico). Primeiro, estimou-se o modelo da equação (4) utilizando o método dos Mínimos Quadrados Ordinários com dados empilhados (pooled $O L S$ ), para efeitos de comparação. Os resultados indicam que o índice de desalinhamento da taxa real de câmbio não é significativo (coluna I e II na Tabela 2 do apêndice). 
Tabela 2 - Desalinhamento e Crescimento - MQO (pooled regression) $x$ Efeitos fixos $x$ Efeitos aleatórios

\begin{tabular}{|c|c|c|c|c|c|c|}
\hline \multirow{2}{*}{$\begin{array}{c}\text { Variável } \\
\text { dependente: } \\
\text { TGROWTH }\end{array}$} & \multicolumn{2}{|c|}{$\begin{array}{l}\text { MQO com dados } \\
\text { Empilhados (robust) }\end{array}$} & \multicolumn{2}{|c|}{$\begin{array}{l}\text { Dados em Painel } \\
\text { (Efeito Fixo) }\end{array}$} & \multicolumn{2}{|c|}{$\begin{array}{l}\text { Dados em Painel } \\
\text { (Efeito Aleatório) }\end{array}$} \\
\hline & $\begin{array}{l}\text { Amostra } \\
\text { ampla } \\
\text { (I) }\end{array}$ & $\begin{array}{l}\text { Amostra } \\
\text { restrita } \\
\text { (II) }\end{array}$ & $\begin{array}{l}\text { Amostra } \\
\text { ampla } \\
\text { (III) }\end{array}$ & $\begin{array}{c}\text { Amostra } \\
\text { restrita } \\
\text { (IV) }\end{array}$ & $\begin{array}{c}\text { Amostra } \\
\text { ampla } \\
(\mathrm{V})\end{array}$ & $\begin{array}{l}\text { Amostra } \\
\text { restrita } \\
\text { (VI) }\end{array}$ \\
\hline UNDERVAL & $\begin{array}{c}-0.0000552 \\
(-0.07)\end{array}$ & $\begin{array}{l}0.0000610 \\
(0.06)\end{array}$ & $\begin{array}{c}0.0343 * * * \\
(4.45)\end{array}$ & $\begin{array}{c}0.0246 * * \\
(2.68)\end{array}$ & $\begin{array}{l}0.000521 \\
\quad(0.40)\end{array}$ & $\begin{array}{l}0.000165 \\
(0.10)\end{array}$ \\
\hline$I C E$ & $\begin{array}{c}-0.00686 * * * \\
(-4.47)\end{array}$ & $\begin{array}{c}-0.00527 * \\
(-2.13)\end{array}$ & $\begin{array}{c}0.00407 \\
(0.79)\end{array}$ & $\begin{array}{c}0.0122 * \\
(2.01)\end{array}$ & $\begin{array}{c}-0.00621^{*} \\
(-2.44)\end{array}$ & $\begin{array}{c}-0.00209 \\
(-0.56)\end{array}$ \\
\hline$I N F$ & $\begin{array}{c}-0.00498 \\
(-0.29)\end{array}$ & $\begin{array}{c}-0.00722 \\
(-0.39)\end{array}$ & $\begin{array}{c}-0.0606 * * * \\
(-4.37)\end{array}$ & $\begin{array}{c}-0.0636 * * * \\
(-4.16)\end{array}$ & $\begin{array}{c}-0.0305^{*} \\
(-2.36)\end{array}$ & $\begin{array}{c}-0.0339 * \\
(-2.37)\end{array}$ \\
\hline INVEST & $\begin{array}{c}0.118 * * * \\
(7.65)\end{array}$ & $\begin{array}{c}0.103 * * * \\
(5.97)\end{array}$ & $\begin{array}{c}0.113 * * * \\
(7.05)\end{array}$ & $\begin{array}{c}0.0674 * * * \\
(3.48)\end{array}$ & $\begin{array}{c}0.118 * * * \\
(8.17)\end{array}$ & $\begin{array}{c}0.0852 * * * \\
(4.89)\end{array}$ \\
\hline$E D U C$ & $\begin{array}{c}-0.779 * * * \\
(-3.67)\end{array}$ & $\begin{array}{c}-0.817 * * \\
(-3.15)\end{array}$ & $\begin{array}{l}2.741 \\
(1.63)\end{array}$ & $\begin{array}{l}2.972 \\
(1.31)\end{array}$ & $\begin{array}{c}-0.849 * * * \\
(-3.38)\end{array}$ & $\begin{array}{c}-0.888 * * \\
(-2.71)\end{array}$ \\
\hline KAOPEN & $\begin{array}{c}-0.396 * * * \\
(-4.71)\end{array}$ & $\begin{array}{c}-0.327 * * * \\
(-3.42)\end{array}$ & $\begin{array}{l}-0.480 * \\
(-2.36)\end{array}$ & $\begin{array}{c}-0.729 * * \\
(-3.20)\end{array}$ & $\begin{array}{c}-0.444 * * * \\
(-3.75)\end{array}$ & $\begin{array}{c}-0.442 * * \\
(-3.09)\end{array}$ \\
\hline OPENTRADE & $\begin{array}{l}0.0121 * * * \\
\quad(6.07)\end{array}$ & $\begin{array}{c}0.0120 * * \\
(2.96)\end{array}$ & $\begin{array}{c}0.0190 * \\
(2.11)\end{array}$ & $\begin{array}{c}0.0312 * \\
(2.57)\end{array}$ & $\begin{array}{c}0.0136 * * * \\
\quad(4.13)\end{array}$ & $\begin{array}{c}0.0180 * * \\
(2.81)\end{array}$ \\
\hline COSTANT & \begin{tabular}{|c}
$0.0213 * * *$ \\
$(3.33)$ \\
\end{tabular} & $\begin{array}{c}0.0292 * * * \\
(4.07)\end{array}$ & $\begin{array}{l}-0.0725 \\
(-1.57)\end{array}$ & $\begin{array}{c}-0.0618 \\
(-1.16)\end{array}$ & $\begin{array}{c}0.0334 * * * \\
\quad(4.11)\end{array}$ & $\begin{array}{c}0.0365 * * * \\
\quad(3.31)\end{array}$ \\
\hline \multicolumn{2}{|c|}{$\begin{array}{l}\text { Teste para escolha do } \\
\text { modelo apropriado }\end{array}$} & \multicolumn{2}{|c|}{ Amostra ampla } & \multicolumn{2}{|c|}{ Amostra restrita } & Decisão \\
\hline $\begin{array}{c}\text { F-test } \\
\text { Prob > F }\end{array}$ & & \multicolumn{2}{|c|}{$\begin{array}{c}4.03 \\
0.00000\end{array}$} & \multicolumn{2}{|c|}{4.33} & $\begin{array}{l}\text { Efeito } \\
\text { Fixo }\end{array}$ \\
\hline \multicolumn{2}{|c|}{$\begin{array}{l}\text { Breusch e Pagan (valor } \chi^{2} \text { ) } \\
\text { Prob }>\chi^{2}\end{array}$} & \multicolumn{2}{|c|}{$\begin{array}{c}129.06 \\
0.00000\end{array}$} & \multicolumn{2}{|c|}{$\begin{array}{c}97.26 \\
0.00000\end{array}$} & $\begin{array}{l}\text { Efeito } \\
\text { Aleatório }\end{array}$ \\
\hline \multicolumn{2}{|c|}{ Hausman (valor $\chi^{2}$ ) } & \multicolumn{2}{|c|}{57.78} & \multicolumn{2}{|c|}{34.64} & $\begin{array}{l}\text { Efeito } \\
\text { Fixo }\end{array}$ \\
\hline
\end{tabular}

Nota: $* \mathrm{p}<0.05, * * \mathrm{p}<0.01, * * * \mathrm{p}<0.001$. Entre parênteses os valores da estatística $t$. (i) Estimativas MQO pooled já corrigidas para potencial heterocedasticidade; (ii) F-Teste: $H_{0}$ : todos os erros são independentes e identicamente distribuídos (iid); (iii) Teste Breusch e Pagan: $H_{0}$ : Os erros do modelo de efeitos aleatórios são iid; (iv) Teste de Hausman: $H_{0}$ : A diferença nos coeficientes dos modelos testados é não sistemática. 
Convém ressaltar que esse modelo admite por hipótese a exogeneidade contemporânea das variáveis explicativas, o que implica que os regressores não podem ser correlacionados com o erro idiossincrático no mesmo período. Contudo, essa condição - necessária para consistência desse estimador - pode não ser observada como consequência da omissão de variáveis relevantes no modelo de regressão. Uma forma de resolver esse problema é utilizar dados em painel mediante a consideração explícita de efeitos individuais não observados, em que sua identificação se torna possível quando a dimensão temporal é incorporada à análise. Nesse sentido, investigamos a seguir a relação de interesse utilizando a técnica de dados em painel.

Os resultados da estimação para o modelo com dados em painel com EF e EA são reportados nas colunas III-VI, respectivamente. Observa-se que o índice desalinhamento cambial apresentou sinal esperado (e significativo) nas estimativas por EF. Com base nesses resultados, rejeita-se a hipótese nula de que o desalinhamento da taxa real de câmbio não tem efeitos sobre a taxa de crescimento, ou seja, as evidências sugerem que esse efeito existe e é positivo.

O coeficiente associado ao desalinhamento da taxa real de câmbio é igual a 0,034 para a amostra ampla e 0,024 para a amostra restrita, valores altamente significativos. Portanto uma subvalorização da taxa real de câmbio tem efeitos significativos na taxa de crescimento do produto, considerando a amostra ampla e reduzida, respectivamente. Ou ainda, os resultados mostram que em ambos os casos uma política baseada na manutenção de uma taxa real de câmbio subvalorizada impulsionaria o crescimento econômico.

Em termos das variáveis de controle, o índice ICE apresentou sinal esperado (positivo) e com nível de significância de 5\% para amostra restrita, reafirmando a importância da taxa real de câmbio para o crescimento. Porém a mesma amostra não foi significativa para a amostra ampla. Esse resultado está de acordo com os resultados alcançados por Gabriel e Missio (2018), para os quais a subvalorização da taxa real de câmbio afeta de maneira positiva a produção de países em desenvolvimento ou emergentes. Ou seja, mesmo após controlar pelo grau de complexidade econômica, ainda assim os efeitos de uma RER competitiva são positivos sobre a taxa de crescimento do produto, especialmente pela sua influência sobre as indústrias manufatureiras. 
A variável que capta a dimensão da educação formal não foi estatisticamente significativa para nenhuma das amostras. Uma interpretação possível para o resultado é o fato de a capacidade de produzir de um país e, portanto, de crescer, pode estar além do conhecimento formal. Uma possível explicação é o reduzido horizonte temporal do exercício realizado (painel curto), ou seja, a educação tende a ser uma importante variável para o crescimento no longo prazo.

Hausmann et al. (2014) fazem uma comparação entre o índice de complexidade e de educação:

Para uma economia complexa existir, seus membros devem ser capazes de ler, escrever e manipular símbolos, como números ou funções matemáticas. Porém as habilidades adquiridas na escola podem ser uma fraca proxy para o conhecimento produtivo da sociedade (p. 34).

Enquanto as medidas de educação utilizam dados da educação formal, o índice de complexidade enfatiza o conhecimento produtivo tácito que está incorporado nas atividades econômicas de um país.

Os coeficientes obtidos (sinal negativo) para a abertura financeira vão ao encontro dos argumentos pós-keynesianos (ver Meyrelles Filho; Jayme Junior, 2009; Barbosa et al., 2018), demonstrando a importância de se ter certa restrição ao grau de abertura financeira principalmente para os países emergentes. $\mathrm{O}$ argumento é que esses países são suscetíveis à entrada massiva de recursos que podem sobrevalorizar suas taxas de câmbio. Uma sobrevalorização sistemática do câmbio exerce efeitos negativos sobre as exportações líquidas, impactando negativamente $o$ emprego e o nível de atividade interna.

Por fim, como esperado, os coeficientes associados à variável inflação são negativos e fortemente significativos do ponto de vista estatístico; enquanto o grau de abertura e a taxa de investimento afetam positivamente a taxa de crescimento do produto.

Todas as estimativas incluem dummy de tempo para cada período com o intuito de capturar mudanças mundiais que afetassem todos os países. Aplicou-se, também, o teste de raiz unitária de Levin, Lin e Chu (2002). Os resultados rejeitam a hipótese nula ( $H_{0}$ : não estacionaridade das séries individuais).

Para garantir robustez aos resultados encontrados, seguindo Missio et al. (2015), foram utilizados os testes de Wald modificado para heterocedasticidade em modelos de regressão com efeitos fixos e o teste de Wooldridge para testar a 
presença de autocorrelação no modelo em painel. Os resultados dos testes indicaram que os erros do modelo são autocorrelacionados e heterocedásticos. Para corrigir esses problemas, foi feita a estimação utilizando o método de Mínimos Quadrados Generalizados (MQG), que corrige o problema da heterocedasticidade. Em segundo, estimou-se o modelo de efeitos fixos (within), corrigindo para o problema da autocorrelação quando o erro idiossincrático é autorregressivo de primeira ordem. Em terceiro, estimou-se com as correções de Driscoll e Kraay (1998) para os erros padrões dos coeficientes estimados por efeitos fixos. A estrutura do erro idiossincrático é assumida como sendo heterocedástica, autocorrelacionada e, possivelmente, correlacionada entre os grupos (painéis). Nesse caso, os erros padrões são robustos a várias formas de dependência transversal ("espacial") e temporal (quando a dimensão do tempo se torna grande). 
Tabela 3 - Teste de Robustez

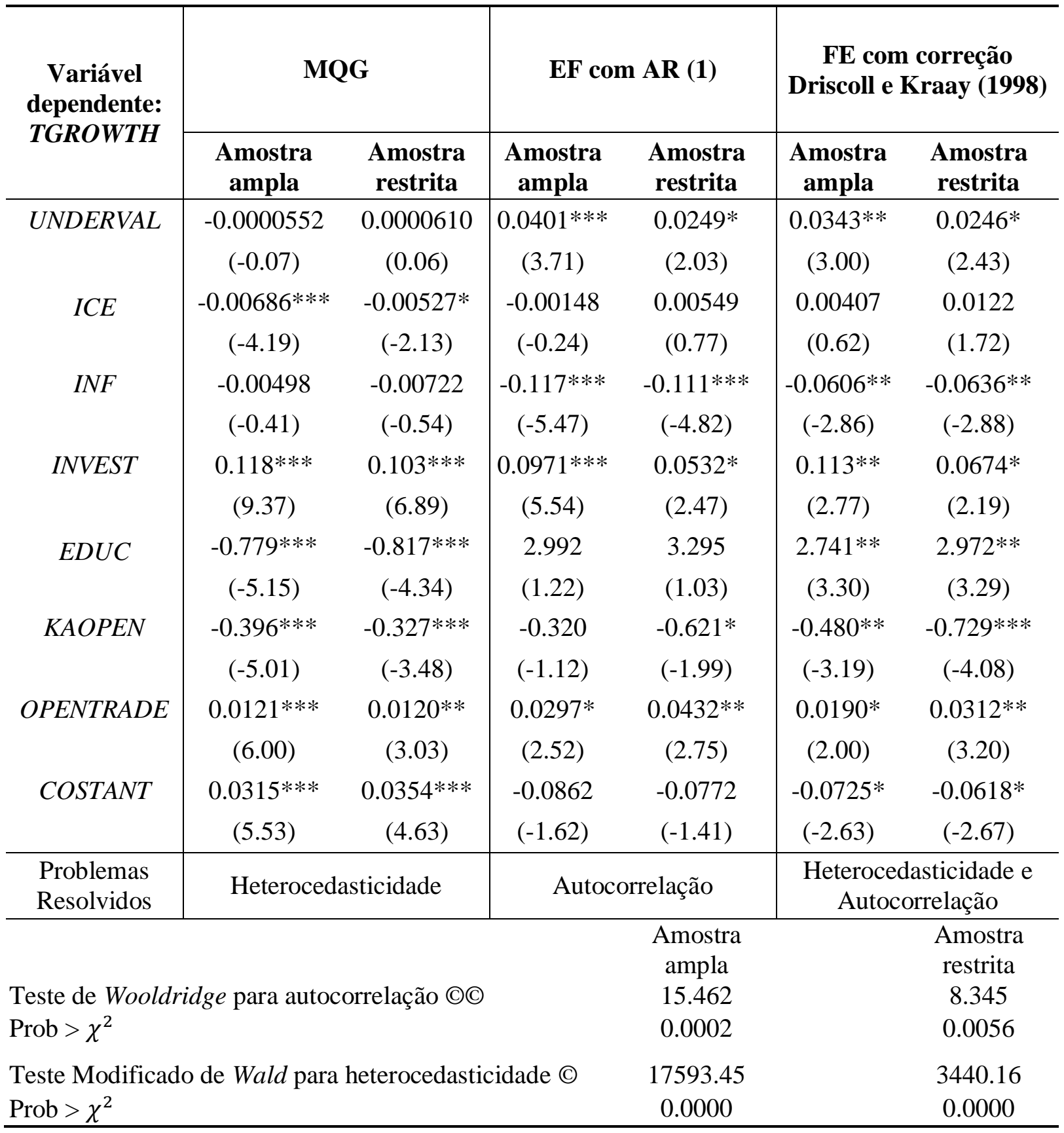

Nota: $* \mathrm{p}<0.05, * * \mathrm{p}<0.01 * *, * * * \mathrm{p}<0.0001$. AR refere-se à autocorrelação de primeira ordem. Entre parêntesis os valores da estatística $t$. Em todas as estimativas foram incluídas as variáveis dummys temporais. ()( Teste para autocorrelação em dados em painel - Hipótese $H_{0}$ : Inexiste autocorrelação de primeira ordem. (C) Teste para heterocedasticidade em modelo de regressão de efeito fixo - Hipótese $H_{0}$ : sigma $(i)^{2}=\operatorname{sigma}^{2}$ para todo $i$.

Com a correção dos problemas de heterocedasticidade e autocorrelação, as estimativas dos coeficientes de interesse do modelo proposto, em geral, mantêm o sinal esperado e são estatisticamente significativas. Isso implica que permanecem 
válidas as relações esperadas entre a variável dependente e as variáveis explicativas, demonstrando a robustez dos resultados encontrados.

\section{Considerações finais}

Os resultados apresentados para a amostra dos países em desenvolvimento, durante o período de 2001 a 2014, corroboram os argumentos apresentados pela literatura neste presente trabalho, segundo os quais a subvalorização da taxa real de câmbio favorece o crescimento econômico. Ademais, os resultados alcançados mostram que a subvalorização da taxa real de câmbio também pode ter efeitos positivos sobre a taxa de crescimento econômico das economias avançadas.

A variável de controle, o índice de complexidade, como proxy para o progresso tecnológico, não teve significância estatística para a amostra ampla. Uma possível explicação para tal resultado advém do entendimento de que, para as economias avançadas, o progresso técnico tende a ser mais diversificado, o que, somado ao maior desenvolvimento do Sistema Nacional de Inovação, faz com que o progresso tecnológico seja muito mais resultado da própria atividade produtiva do que dependente de estímulos advindos da política cambial. Isso tende a não ser verdadeiro nos países em desenvolvimento que, por estarem distantes da fronteira tecnológica, dependem de estímulos advindos da condução da política econômica (cambial).

Em resumo, a condução da política cambial no sentido de manter uma taxa de câmbio estável e competitiva pode ser um estimulo importante para os países em desenvolvimento. Vale ressaltar que o Banco Central tem uma variedade de medidas à sua disposição para influenciar a taxa real de câmbio, que, no mundo atual, é fundamentalmente determinado pelo movimento de capitais. Dentre elas, destacam-se as intervenções esterilizadas e o controle de capitais.

Nesse contexto, quando se considera que o BC pode intervir na dinâmica cambial, a ponto de influenciar a determinação do nível da taxa real de câmbio por tempo suficiente para que os efeitos sobre a economia ocorram (como a mudança estrutural, por exemplo), então essa política passa a ser fundamental para o crescimento, sobretudo para os países em desenvolvimento. 


\section{Referências}

AHMED, S.; APPENDINO, M.; RUTA, M. Global value chains and the exchange rate elasticity of exports. The BE Journal of Macroeconomics, v. 17, n. 1, p. 1-28, 2016.

ARAUJO, E., GALA, P. Regimes de crescimento econômico no Brasil: evidências empíricas e implicações de política. Estudos Avançados, v. 25, n. 75, p. 41-56, 2012.

BALTAR, C.; HIRATUKA, C.; LIMA, G. Real exchange rate and investment in the Brazilian manufacturing industry. Journal of Economic Studies, v. 43, n. 2, p. 288-308, 2016.

BARBOSA-FILHO, N. Exchange rates, growth and inflation. In: Annual Conference on Development and Change, Campos do Jordão, Brazil. 2006. p. 17-20.

BARBOSA, L.; JAYME JUNIOR, F.; MISSIO, F. Managing real exchange rate for economic growth: empirical evidences from developing countries. Journal of Post Keynesian Economics, v. 41, n. 4 p. 598-619, 2018.

BASTOS, F., WANG, K. Long-run growth in Latin America and the Caribbean: the role of economic diversification and complexity. Regional Economic Outlook, p. $67-77,2015$.

BLECKER, R. The debate over 'Thirlwall's law': balance-of-paymentsconstrained growth reconsidered. European Journal of Economics and Economic Policies: Intervention, v. 13, n. 3, p. 275-290, 2016.

BOGGIO, L.; BARBIERI, L. International competitiveness in post-Keynesian growth theory: controversies and empirical evidence. Cambridge Journal of Economics, v. 41, n. 1, p. 25-47, 2017.

BORDO, M.; CHOUDHRI, E.; FAZIO, G.; MACDONALD, R. The real exchange rate in the long run: Balassa-Samuelson effects reconsidered. Journal of International Money and Finance, v. 75, p. 69-92, 2017.

BRESSER-PEREIRA, L. C.; OREIRO, J. L.; MARCONI, N. Macroeconomia desenvolvimentista: teoria e política econômica do novo desenvolvimentismo. Rio de Janeiro: Elsevier, 2017.

BREUSCH, T.; PAGAN, A. The Lagrange multiplier test and its applications to model specification in econometrics. The review of economic studies, v. 47, n. 1, p. 239-253, 1980. 
CIMOLI, M.; FLEITAS, S.; PORCILE, G. Technological intensity of the export structure and the real exchange rate. Economics of innovation and new technology, v. 22, n. 4, p. 353-372, 2013.

CHEN, S.-S. Exchange rate undervaluation and R\&D activity. Journal of International Money and Finance, v. 72, n. C, p. 148-160, 2017.

COMUNALE, M. Dutch disease, real effective exchange rate misalignments and their effect on GDP growth in EU. Journal of International Money and Finance, v. 73, n. B, p. 350-370, 2017.

CLARK, C. The conditions of economic progress. The conditions of economic progress, 1967.

DEMIRAL, M. Knowledge, complexity and economic growth: multi-country evidence by development stages. Journal of Knowledge Management, Economics and Information Technology, v. VI, n. 1, p. 1-27, 2016.

DENISON, E. Why growth rates differ: postwar experience in nine western countries. Washington: The Brookings Institution, 1967.

DI NINO, V.; EICHENGREEN, B.; SBRACIA, M. Real exchange rates, trade, and growth. In: TONIOLO, G. The Oxford Handbook of the Italian Economy Since Unification. Oxford: Oxford University Press, 2013.

DRISCOLL, J.; KRAAY, A. Consistent covariance matrix estimation with spatially dependent panel data. Review of Economics and Statistics, v. 80, n. 4, p. 549-560, 1998.

EICHENGREEN, B. The real exchange rate and economic growth. The World Bank, Commission on Growth and Development Working Paper n ${ }^{\circ}$ 4, 2008.

EL-SHAGI, M.; LINDNER, A.; VON SCHWEINITZ, G. Real effective exchange rate misalignment in the euro area: a counterfactual analysis. Review of International Economics, v. 24, n. 1, p. 37-66, 2016.

FERRARI, M.; FREITAS, F.; BARBOSA FILHO, N. A taxa de câmbio real e a restrição externa: uma proposta de releitura com elasticidades endógenas. Brazilian Journal of Political Economy, v. 33, n. 1, p. 60-81, 2013.

FRENKEL, R.; RAPETTI, M. The real exchange rate as a target of macroeconomic policy. MPRA Paper 59335, University Library of Munich, Germany, 2014. 
FREUND, C.; PIEROLA, M. Export surges. Journal of Development Economics, v. 97 , n. 2, p. 387-395, 2012.

GABRIEL, L.; JAYME JUNIOR, F.; OREIRO, J. L. A North-South model of economic growth, technological gap, structural change and real exchange rate. Structural Change and Economic Dynamics, v. 38, p. 83-94, 2016.

GABRIEL, L.; MISSIO, F. Real exchange rate and economic complexity in a North-South structuralist BoPG model. PSL Quarterly Review, v. 71, n. 287, p. 439-465, 2018.

GALA, P. Real exchange rate levels and economic development: theoretical analysis and econometric evidence. Cambridge Journal of Economics, v. 32, n. 2 , p. 273-288, 2008.

GOUVEA, R.; LIMA, G. Balance-of-payments-constrained growth in a multisectoral framework. Journal of Economic Studies, v. 40, n. 2, p. 240-254, 2013.

GUZMAN, M.; OCAMPO, J.; STIGLITZ, J. Real exchange rate policies for economic development. World Development, v. 110, p. 51-62, 2018.

GLÜZMANN, P.; LEVY-YEYATI, E.; STURZENEGGER, F. Exchange rate undervaluation and economic growth: Díaz Alejandro (1965) revisited. Economics Letters, v. 117, n. 3, p. 666-672, 2012.

HABIB, M.; MILEVA, E.; STRACCA, L. The real exchange rate and economic growth: revisiting the case using external instruments. Journal of International Money and Finance, v. 73, p. 386-398, 2017.

HAUSMAN, J. Specification tests in econometrics. Econometrica, v. 46, n. 6, p. 1251-1271, 1978.

HAUSMANN, R.; HIDALGO, C. The network structure of economic output. Journal of Economic Growth, v. 16, n. 4, p. 309-342, 2011.

HAUSMANN, R.; HILDAGO, C. (Orgs.). The atlas of economic complexity: Mapping paths to prosperity. Cambridge: The MIT Press, 2014.

KALDOR, N. Causes of the slow rate of economic growth of the United Kingdom: an inaugural lecture. Cambridge: Cambridge University Press, 1966.

KALDOR, N. Strategic factors in economic development. Ithaca: Cornell University Press, 1967. 
KALDOR, N. The case for regional policies. Scottish Journal of Political Economy, v. 17, n. 3, p. 337-348, 1970.

KUZNETS, S.; MURPHY, J. Modern economic growth: rate, structure, and spread. New Haven: Yale University Press, 1966.

KUZNETS, S. Growth and structural shifts. Economic growth and structural change in Taiwan: The postwar experience of the Republic of China, p. 15-131, 1979.

LEVIN, A.; LIN, C.; CHU, C. Unit root tests in panel data: asymptotic and finitesample properties. Journal of Econometrics, v. 108, n. 1, p. 1-24, 2002.

LEWIS, W. Economic Development with Unlimited Supply of Labour. The Manchester School, v. 22, p. 139-191, 1954.

MCCOMBIE, J., ANGERIZ, A.; ROBERTS, M. Increasing returns and growth in industries in the EU regions: paradoxes and conundrums. Spatial Economic Analysis, v. 4, n. 2, p. 147-168, 2009.

MARCONI, N.; ARAUJO, E.; OREIRO, J. The exchange rate, income elasticities, and structural change: theoretical foundations and empirical evidence. In: The Conference of the Research Network Macroeconomics and Macroeconomic Policies. 2015.

MCMILLAN, M.; RODRIK, D. Globalization, structural change and productivity growth. National Bureau of Economic Research, p. 1-54, 2011.

MEYER, D. Structural changes in the economy of Hong Kong since 1997. China Review, v. 8, n. 1, p. 7-29, 2008.

MEYRELLES FILHO, S.; JAYME, JR., F. Mobilidade de capitais e crescimento econômico: uma análise empírica dinâmica com dados em painel. Revista EconomiA, v. 10, n. 4, p. 789-815, 2009.

MISSIO, F.; JAYME JUNIOR, F. Structural heterogeneity and endogeneity of elasticities on the balance of payments constrained growth model. In: SOUKIAZIS, E.; CERQUEIRA, P. (Orgs.). Models of balance of payments constrained growth: history, theory and empirical evidence. Londres: Palgrave Macmillan, p. 239-267, 2012.

MISSIO, F.; JAYME JUNIOR, F.; BRITTO, G.; OREIRO, J. Real exchange rate and economic growth: new empirical evidence. Metroeconomica, v. 66, p. 686$714,2015$. 
MISSIO, F.; ARAUJO, R.; JAYME JR, F. Endogenous elasticities and the impact of the real exchange rate on structural economic dynamics. Structural Change and Economic Dynamics, v. 42, n. C, p. 67-75, 2017.

MONTIEL, P. The long-run equilibrium real exchange rate: conceptual issues and empirical research. In: HINKLE, L.; MONTIEL, P. (Org.). Exchange rate misalignment: Concepts and measurement for developing countries. Washington: World Bank, 1999, p. 219-263.

MARTINS NETO, A.; PORCILE, G. Destabilizing austerity: fiscal policy in a BOP-dominated macrodynamics. Structural Change and Economic Dynamics, v. 43, p. 39-50, 2017.

NURKSE, R. Conditions of international monetary equilibrium: international finance section, department of economics and social institutions. Princeton: Princeton University, 1945.

OREIRO, J. L.; PUNZO, L.; ARAÚJO, E.; SQUEFF, G. Taxa real de câmbio, desalinhamento cambial e crescimento econômico no Brasil (1994-2007). Brazilian Journal of Political Economy, v. 31, n. 4, p. 551-562, 2011.

OREIRO, J.; MISSIO, F.; JAYME JR, F. Capital accumulation, structural change and real exchange rate in a Keynesian-structuralist growth model. Panoeconomicus, v. 62, n. 2, p. 237-256, 2015.

RAZMI, A. Correctly analysing the balance-of-payments constraint on growth. Cambridge Journal of Economics, v. 40, n. 6, p. 1581-1608, 2015.

RIBEIRO, R.; MCCOMBIE, J.; LIMA, G. Does real exchange rate undervaluation really promote economic growth? Structural Change and Economic Dynamics, v. 52, p. 408-417, 2020.

ROMERO, J.; BRITTO, G. Increasing returns to scale, technological catch-up and research intensity: endogenising the Verdoorn coefficient. Cambridge Journal of Economics, v. 41, n. 2, p. 391-412, 2017.

RODRIK, D. The real exchange rate and economic growth. Brookings papers on conomic activity, v. 2008, n. 2, p. 365-412, 2008.

SCHROEDER, M. The equilibrium real exchange rate and macroeconomic performance in developing countries. Applied Economics Letters, v. 24, n. 7, p. 506-509, 2017. 
SEKKAT, K. Exchange rate misalignment and export diversification in developing countries. The Quarterly Review of Economics and Finance, v. 59, p. 1-14, 2016.

SYRQUIN, M. Patterns of structural change. In: CHENERY, H.; SRINIVASAN, T. (Orgs.). Handbook of development economics. Amsterdam: North-Holland, 1988, p. 203-273.

STOJKOSKI, V.; KOCAREV, L. The relationship between growth and economic complexity: evidence from Southeastern and Central Europe. MPRA Paper 77837, 2017.

VU, K. M. Structural change and economic growth: empirical evidence and policy insights from Asian economies. Structural Change and Economic Dynamics, v. 41, p. 64-77, 2017.

\section{Financiamento}

Os autores agradecem à Fundação de Amparo à Pesquisa do Estado de Goiás (FAPEG) pelo suporte recebido.

\section{Agradecimentos}

O professor Fabricio José Missio agradece o apoio do Conselho Nacional de Desenvolvimento Científico e Tecnológico - CNPq (bolsa produtividade em pesquisa).

\section{Apêndice}

Tabela 1 - Estatísticas básicas - Amostra ampla

\begin{tabular}{c|c|c|c|c}
\hline Variável & Média & Desvio Padrão & Mínimo & Máximo \\
\hline TGROWTH & 0.0385976 & 0.0407397 & -0.1481416 & 0.345 \\
UNDERVAL & $3.20 \mathrm{E}-09$ & 1.586872 & -6.12912 & 9.399748 \\
ICE & 0.2689998 & 0.9587385 & -2.791360 & 2.611650 \\
INF & 0.0541694 & 0.081716 & -0.03659 & 1.52586 \\
INVEST & 0.2258103 & 0.0918355 & 0.00000 & 0.63476 \\
EDUC & 0.0247809 & 0.0086823 & 0.00000 & 0.037343 \\
KAOPEN & 0.0078187 & 0.015498 & -0.0191043 & 0.0236 \\
OPENTRADE & 0.8796992 & 0.5510827 & 0.1945883 & 4.416038 \\
\hline
\end{tabular}

Tabela 5 - Estatísticas básicas - Amostra restrita

\begin{tabular}{c|c|c|c|c}
\hline Variável & Média & Desvio Padrão & Mínimo & Máximo \\
\hline
\end{tabular}




\begin{tabular}{c|c|c|c|c} 
TGROWTH & 0.0471267 & 0.0407722 & -0.148 & 0.345 \\
UNDERVAL & $-1.61 \mathrm{E}-09$ & 1.686733 & -5.64822 & 9.886981 \\
ICE & -0.2035841 & 0.7095158 & -2.791360 & 1.533970 \\
INF & 0.0688902 & 0.094966 & -0.03659 & 1.52586 \\
INVEST & 0.2244712 & 0.1065892 & 0.00000 & 0.63476 \\
EDUC & 0.0213798 & 0.0083533 & 0.00000 & 0.034112 \\
KAOPEN & 0.0018157 & 0.014648 & -0.0191043 & 0.0236 \\
OPENTRADE & 0.7850282 & 0.3318922 & 0.1945883 & 2.103738 \\
\hline
\end{tabular}

Tabela 6 - Matriz de correlação - Amostra ampla

\begin{tabular}{l|cccccccc}
\hline & TGROWTH & LNUNDERVAL & COMPLEX & INF & INVEST & HUM & KAOPEN OPENTRADE \\
\hline TGROWTH & 1.0000 & & & & & & & \\
UNDERVAL & -0.1154 & 1.0000 & & & & & & \\
ICE & -0.2800 & 0.5424 & 1.0000 & & & & & \\
INF & 0.1176 & 0.0413 & -0.3128 & 1.0000 & & & & \\
INVEST & 0.2509 & 0.0379 & 0.0920 & -0.0485 & 1.0000 & & \\
EDUC & -0.2874 & 0.1052 & 0.5787 & -0.2769 & 0.1072 & 1.0000 & & \\
KAOPEN & -0.2848 & 0.3260 & 0.5456 & -0.3088 & -0.0417 & 0.4399 & 1.0000 & \\
OPENTRADE & 0.1152 & 0.1997 & 0.2306 & -0.0045 & 0.1107 & 0.0762 & 0.1536 & 1.0000 \\
\hline
\end{tabular}

Tabela 7 - Matriz de correlação - Amostra restrita

\begin{tabular}{l|cccccccc}
\hline & TGROWTH & LNUNDERVAL & COMPLEX & INF & INVEST & HUM & KAOPEN OPENTRADE \\
\hline TGROWTH & 1.0000 & & & & & & & \\
LNUNDERVAL & 0.0114 & 1.0000 & & & & & & \\
ICE & -0.1114 & 0.4441 & 1.0000 & & & & & \\
INF & 0.0294 & 0.1866 & -0.1907 & 1.0000 & & & & \\
INVEST & 0.2528 & 0.0324 & 0.1491 & -0.0611 & 1.0000 & & \\
EDUC & -0.1818 & -0.1964 & 0.3641 & -0.1570 & 0.1158 & 1.0000 & & \\
KAOPEN & -0.1454 & 0.0927 & 0.2046 & -0.1794 & -0.0893 & 0.1352 & 1.0000 & \\
OPENTRADE & 0.1210 & 0.2303 & 0.2689 & 0.0603 & 0.1371 & 0.0210 & 0.1304 & 1.0000 \\
\hline
\end{tabular}

